\title{
Effect of a medicinal and edible decoction YH0618 on chemotherapy-induced dermatologic toxicity: a randomized controlled trial
}

\author{
Jieshu You ${ }^{1,2}$, Yanhua He ${ }^{1}$, Hui Zhi ${ }^{3}$, Victor Hofun Lee ${ }^{4,5}$, Suetmui Chan ${ }^{3}$, Lixing Lao ${ }^{1}$, Huanlan Liu ${ }^{1}$, \\ Jianping Chen ${ }^{1,5}$
}

${ }^{1}$ School of Chinese Medicine, The University of Hong Kong, Hong Kong, China; ${ }^{2}$ School of Basic Medical Sciences, Guangzhou University of Chinese Medicine, Guangzhou, China; ${ }^{3}$ Department of Social Work \& Social Administration, The University of Hong Kong, Hong Kong, China; ${ }^{4}$ Department of Clinical Oncology, The University of Hong Kong, Hong Kong, China; ${ }^{5}$ Shenzhen Institute of Research and Innovation, The University of Hong Kong, Shenzhen, China

Contributions: (I) Conception and design: J You, H Zhi, L Lao, J Chen; (II) Provision of study material or patients: J You, Y He, H Lee, S Chan; (III) Collection and assembly of data: Y He, J You; (IV) Data analysis and interpretation: J You, H Zhi, L Lao, H Liu, J Chen; (IV) Manuscript writing: All authors; (VI) Final approval of manuscript: All authors; (VI) Accountable for all aspects of the work: All authors.

Correspondence to: Dr. Jianping Chen. School of Chinese Medicine, The University of Hong Kong, 10 Sassoon Road, Pokfulam, Hong Kong, China. Email: abchen@hku.hk.

Background: Dermatologic toxicities are the common adverse events (AE) with several chemotherapy agents, but they are usually neglected in the research literature and clinical practice, and there are no clinically safe and effective methods to solve the problem. This study was to determine whether a medicinal and edible decoction YH0618 is effective in accelerating reducing chemotherapy-induced dermatologic toxicity in cancer patients who have completed chemotherapy.

Methods: This was a prospective randomized controlled trial conducted between 2015 and 2017. Cancer patients who have completed chemotherapy (received taxanes or anthracyclines or fluoropyrimidine) within two weeks were enrolled and then they were randomly divided into YH0618 decoction group ( $\mathrm{n}=104)$ and wait-list control $(n=110)$. The primary end points were the incidence of protocol-specified grade $\geq 2$ dermatologic toxicities after 6-week intervention assessed using the National Cancer Institute-Common Terminology Criteria for Adverse Events (NCI-CTCAE) Chinese version 4.0, and changes of fingernails color and skin color evaluated by $\mathrm{L}^{*} \mathrm{a}^{*} \mathrm{~b}$ after 6 weeks of intervention. Secondary end points included assessment of quality of life (QOL) and fatigue, and some clinical objective indicators associated with myelosuppression, hepatotoxicity and nephrotoxicity.

Results: The study included 214 participants [mean (SD) age, 52.49 (9.08) years in YH0618 group and 50.44 (9.71) years in wait-list group]. At 6-week, YH0618 significantly reduced the incidence of grade $\geq 2$ in nail discoloration [odds ratio (OR), 0.653; 95\% CI, 0.5-0.9; $\mathrm{P}=0.005$ ] and alopecia (OR, 0.776; 95\% CI, 0.6-1.0; $\mathrm{P}=0.048$ ) compared with control group. Besides, YH0618 increased the $\mathrm{L}^{*}$ value and reduced the $\mathrm{a}^{*}$ and $\mathrm{b}^{*}$ values compared with control group, indicating that YH0618 increased the brightness and reduced hyperpigmentation. YH0618 also significantly reduced chemotherapy-induced fatigue (95\% CI, 0.2-4.8; $\mathrm{P}=0.033$ ).

Conclusions: YH0618 may be a safe method in ameliorating chemotherapy-induced dermatologic toxicity especially nail discoloration, alopecia and skin hyperpigmentation, and on improving fatigue.

Trial registration: The trial was registered in the Chinese Clinical Trials Registry, ChiCTRIOR-15006486.

Keywords: Homology of medicine and food; YH0618 decoction; clinical trial; chemotherapy-induced dermatologic toxicity; hyperpigmentation 
Submitted Jun 07, 2020. Accepted for publication Oct 16, 2020.

doi: 10.21037/atm-20-5181

View this article at: http://dx.doi.org/10.21037/atm-20-5181

\section{Introduction}

Chemotherapy is a standard regimen for cancer treatment, including killing cancer cells and decreasing the risk of recurrence. However, chemotherapy may result in a series of adverse effects, in which dermatologic toxicities are the one of the most common. Dermatologic toxicities are major side effects of more than 30 anticancer agents like antimetabolites, hormonal agents, topoisomeraseinteracting agents and taxanes (1). Specifically, chemotherapy-induced dermatologic toxicities (CIDT) symptoms mainly include dry skin, alopecia, pruritus, bullous dermatitis, pain of skin, paronychia, skin infection, nail discoloration and nail loss. In clinical practice, the main types of dermatologic toxicities caused by different chemotherapeutic drugs are also different. For example, taxane-related nail changes like Beau lines, nail pigmentation, acute paronychia, and splinter hemorrhage, and can occur in up to $88 \%$ of patients (2). Acral erythema is commonly observed in patients receiving capecitabine, with reported rates of $45 \%$ to $56 \%$ in clinical trials (3). Dermatologic toxicities rarely induce fetal events, however, considerable cosmetic concern, pain, infection, functional impairment and further poor quality of life (QOL) will happen.

Recently, various managements are used to prevent CIDT, but there exists no acknowledged and standard methods. Furthermore, success rates with different managements have been variable and usually a device can be only used for deal with one symptom. For instance, scalp cooling is the only method approved by U.S. Food and Drug Administration (FDA) used for chemotherapyinduced hair loss, unfortunately, the success rate of scalp cooling can only reach $50 \%$ (4). Therefore, for clinicians and researchers, they should focus more on CIDT and develop safe and effective ways to reduce CIDT.

YH0618, a medicinal and edible compound prescription, is developed based on the "homology of medicine and food" theory of traditional Chinese Medicine, ancient prescription, and long-term clinical practice. YH0618 contains four medicinal foods, namely Sojae Semen Nigrum (Black Soybean, $30 \mathrm{~g}$ ), Brown Rice (30 g), Black Fungus $(2 \mathrm{~g})$ and Siraitiae Fructus (1 piece), which are recommended by Chinese medical clinicians for cancer patients and all ingredients have a history of safe use. In the long clinical observation, YH0618 soup has been proven to effectively reduce the toxicity caused by chemotherapy, especially alopeica, skin hyperpigmentation and fatigue. The previous animal study showed that YH0618 decoction did not interfere with the anti-tumor effect of chemotherapy drugs (5).

Therefore, the purpose of this clinical trial was to assess whether YH0618 decocotion is effective in accelerating reducing CIDT in cancer patients who have completed chemotherapy (received taxanes or anthracyclines or fluoropyrimidine).

We present the following article in accordance with the CONSORT reporting checklist (available at http://dx.doi. org/10.21037/atm-20-5181).

\section{Methods}

\section{Study design and setting}

The trial was a randomized, assessor-blinded, controlled study conducted from 2015 to 2017, for cancer patients completed chemotherapy. This trial was conducted at the clinics of School of Chinese Medicine, the University of Hong Kong (HKU). The study was conducted in accordance with the Declaration of Helsinki (as revised in 2013). The study was approved by the Institutional Review Board of the University of Hong Kong/Hospital Authority Hong Kong West Cluster (HKU/HA HKW IRB) (No.: UW 15-023) and informed consent was taken from all the patients. In the trial, a Data and Safety Monitoring Board (DSMB) was organized, which is an independent expert group that advises funding agencies and research investigators.

Changes to the clinical trial protocol (6) included expanding inclusion criteria that patient received fluoropyrimidine were also recruited fluoropyrimidine are the main chemotherapy agents causing skin toxicity, such as alopecia, hyperpigmentation, and hand-foot syndrome; recruiting 214 subjects instead of 236, because 236 was calculated based on a $30 \%$ dropout rate; deleting the assessment of tongue color, as the camera could not 
objectively and accurately get the color of tongue.

\section{Participants}

The main eligibility criteria for inclusion were patients with any cancer types and stages aged between $18-75$ years. They completed chemotherapy no more than 2 weeks; had received chemotherapeutic agents containing taxanes or anthracyclines or fluoropyrimidine; and life expectancy was at least 6 months. The main exclusion criteria included subjects receiving detox treatment within 3 months; having any serious mental illness or history, or taking psychotropic drugs; a medical history of dermatosis; severe liver and kidney damage; pregnancy or potential pregnancy; and allergic to certain special foods (such as black soybean). Eligible patients were invited to participate in this study after obtaining their written consent form. All participants were closely monitored during the study. These patients could receive conventional usual care as an additional safeguard.

\section{Randomization and intervention}

The 1:1 random allocation was performed using a computer-generated random code by an independent researcher who did not participate in enrollment. Nontransparent envelopes with the allotted sequences inside were prepared and kept sealed. Because the trial compared the effects of the YH0618 decoction and the wait-list group, it was unable to blind the patients. Thus, the patients knew which group they were in. However, the randomization sequence and different groups were kept hidden from clinicians, data collectors and statisticians.

Prior to intervention, baseline data were collected including demographics, chemotherapy-induced dermatologic toxicity, skin color, nail color, scores of QOL and fatigue, and the indicators of hematotoxicity, hepatotoxicity and nephrotoxicity in the blood. After that, participants assigned to YH0618 group received YH0618 decoction $(1,000 \mathrm{~mL} /$ day $) 6$ days a week for 6 consecutive weeks, followed by uncontrolled follow-up for 6 weeks, while those in wait-list group were asked to wait 6 weeks before receiving YH0618 decoction for 6 weeks. All participants were assessed before the intervention (baseline), and 3, 6 and 12 weeks after intervention. There are no contraindications between $\mathrm{YH} 0618$ and chemotherapy, as all the ingredients are medicinal foods which people often consume in their daily life.
All YH0618 materials meet the food safety requirements of the Food and Environmental Hygiene Department and have obtained quality certificates. The packaging was performed at the clinics of School of Chinese Medicine, HKU. Professional research assistant taught these subjects how to cook and take the YH0618 decoction, including water amount, decoction time and other matters need attention (Appendix I). Quality and compliance of intervention were achieved by checking attendance records and the diary of self-recording form (Appendix II). Patients randomized to YH0618 group who did not receive at least one dose of YH0618 were eliminated.

\section{Outcome measures}

The primary outcomes were the incidence of specific grade $\geq 2$ dermatologic toxicities and changes in nail color, skin color and tongue color at 6 weeks after the intervention, which were performed according to the National Cancer Institute-Common Terminology Criteria for Adverse Events (NCI-CTCAE) Chinese version 4.0 and evaluated respectively by $L^{*} a * b$.

\section{Grading of dermatologic toxicity}

NCI CTCAE is commonly used to monitor and assess the severity of toxicity caused by chemotherapy (7). The CTCAE shows grades 1 to 5 with unique clinical descriptions of severity for each adverse event (AE). Protocol-defined dermatologic toxicity included dry skin, pruritus, skin ulceration, rash acneiform, rash maculopapular, pain of skin, skin hyperpigmentation, palmarplantar erythrodysesthesia syndrome, scalp pain, alopecia, paronychia, nail discoloration, nail ridging and nail loss, which were detailed in Appendix III.

\section{Skin and nail color measurement}

$\mathrm{L}^{*} \mathrm{a}$ b, determined by International Commission on Illumination (CIE) in 1976 is a "gold standard" for evaluating color (8). Value $\mathrm{L}^{*}$ stands for light/dark, ranging from 0 (black) to 100 (white). Value $\mathrm{a}^{*}$ represents red/ green axis, and Value $b^{*}$ represents yellow/blue axis (9). In this study, digital image analysis was used, which not direct contact with the skin and therefore does not interfere with the measurement of skin color (e.g., by blanching the skin). A professional digital camera (G11, Canon Camera Co., Inc., Japan) with manual white balance, 1/60 shutter, 
5.0 aperture, 200 ISO, raw file was used (Figure 1). Digital camera and the environment like light source, location, background, distance between camera and subject were strictly fixed, which was shown in Figure 2. To reduce the interference from external environment, a calibration color chart (Spyder CHECKR 24) was used. Spyder CHECKR24 is composed of a double-sided reference card with 24 color patches on one side (Figure $3 A$ ) and six greyscale tones plus a larger mid-grey patch on the other side (Figure $3 B$ ). It is an accepted and useful color reference tool used to calibrate any kind of camera. Then, color sampler tool in Adobe PhotoShop Creative Suite 6 (CS6) was adopted for

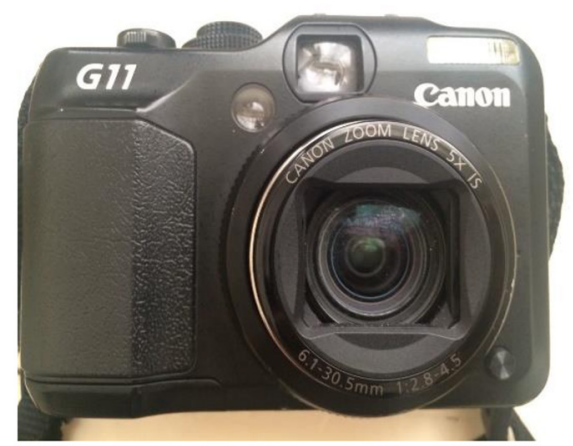

Figure 1 Camera.
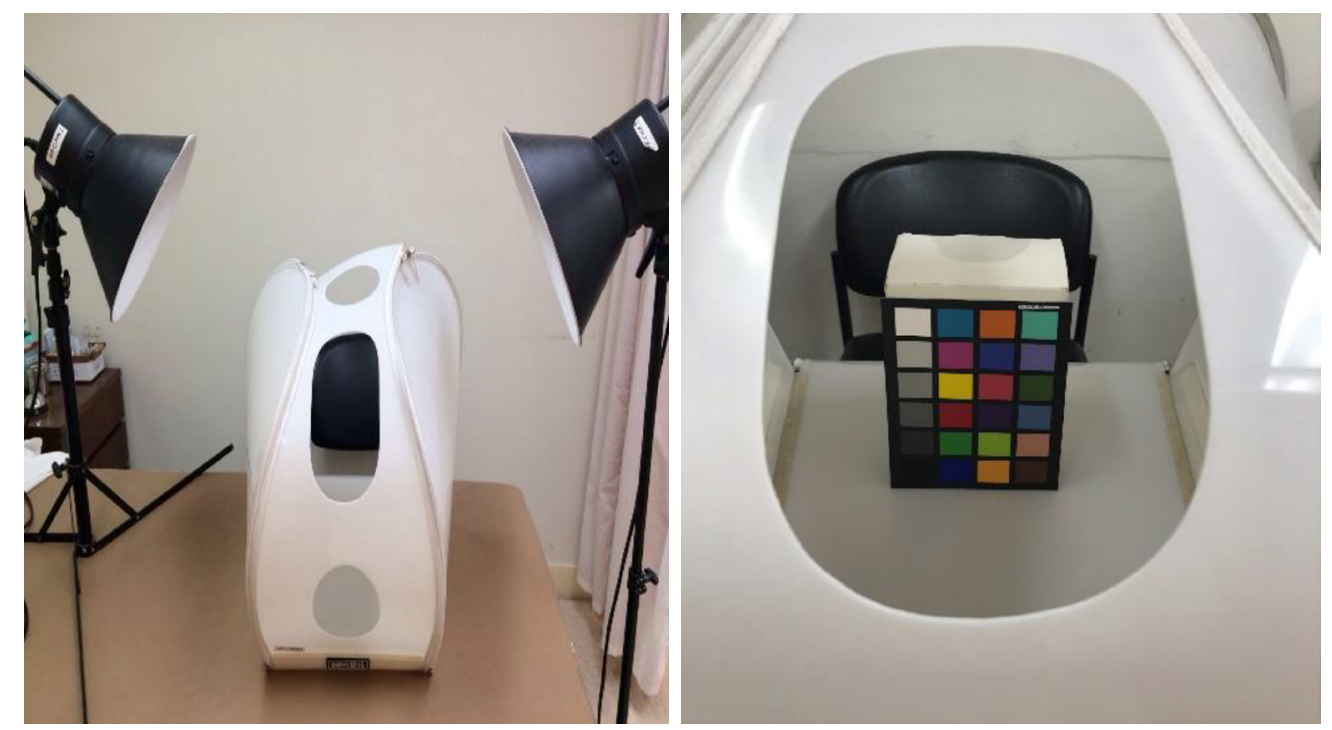

Figure 2 Photography environment.

digital image color analysis, which could retrieve and record $\mathrm{L}^{*} \mathrm{a}^{*} \mathrm{~b}^{*}$ color values. Skin color of the forehead, right and left cheeks, jaw, dorsum of the right and left hands and nail color of ten fingernails were recorded.

Secondary outcomes were evaluated using several instruments. QOL has been regarded as an important indicator for predicting and monitoring the outcome of treatment for cancer patients. Chinese version of the FACT-G instrument with good reliability and validity was used to assess cancer-specific QOL. The tool includes 27 items, which are classified into four domains: Physical Well-Being, Social/Family Well-Being, Emotional WellBeing and Functional Well-Being. A total score was calculated by summing all subscale scores. Higher total scores mean better general QOL. Fatigue was measured by the Chinese version of FACIT-Fatigue version 4 (a 13item FACIT Fatigue Scale), which can be used for any type tumor patients (10). Each item was scored on a 5-point Likert self-reporting scale ranging from 0 to 4 . A total score was obtained by summing all item scores and a high score indicated less fatigue. Clinical objective examination included white blood cells (WBC), hemoglobin (HGB) and platelets (PLT) reflecting myelosupression; alanine aminotransferase (ALT), aspartate transaminase (AST), total protein and albumin (Alba) reflecting hepatotoxicity; and blood urea nitrogen (BUN) and creatinine representing 

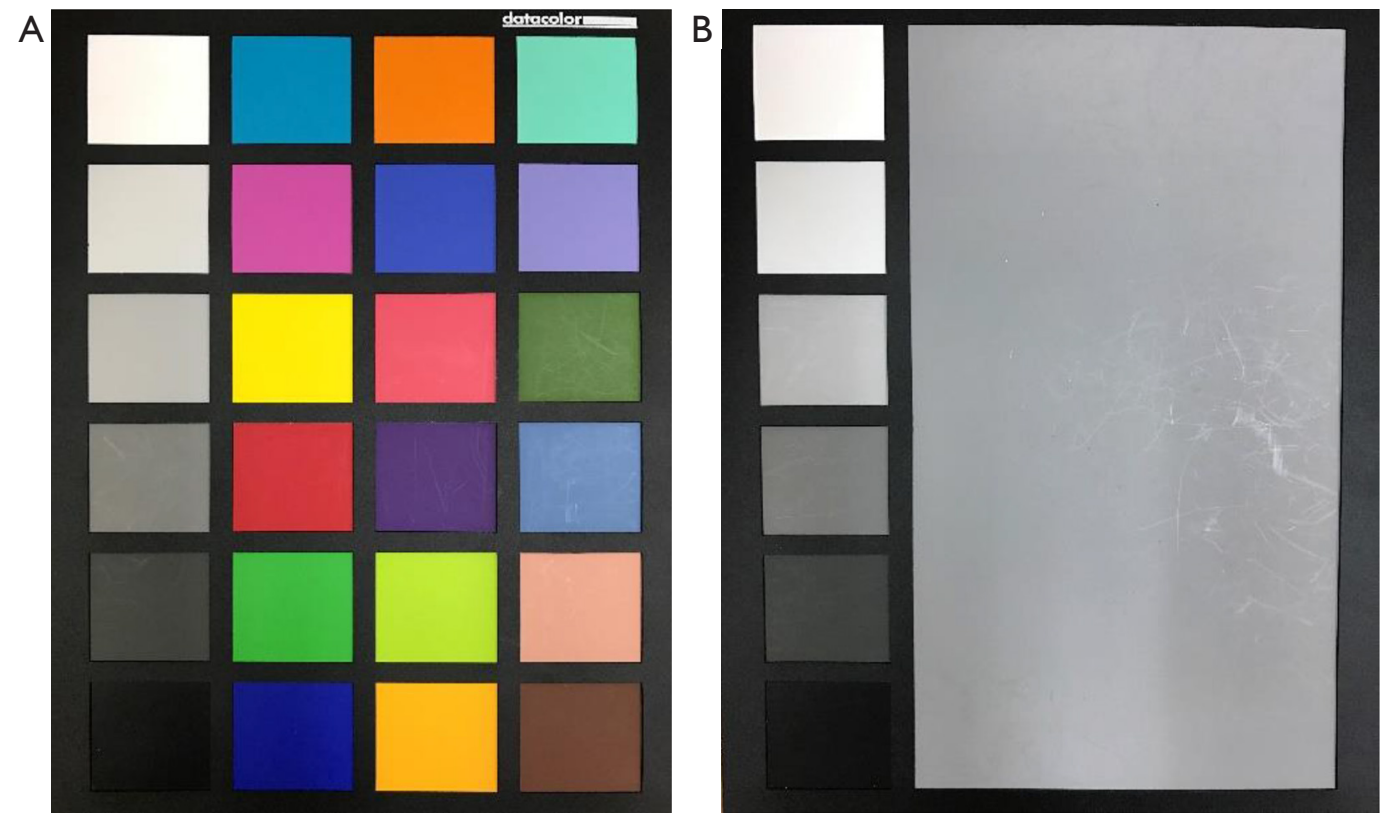

Figure 3 Spyder CHECKR24.

nephrotoxicity.

AE were reported spontaneously by patients and observed by evaluators. AE were graded on a five level (Mild, Moderate, Severe, Life threatening and causing death). When an AE occurs, the researcher took all necessary and appropriate ways to ensure the safety of the patients. Any issues related to the intervention-related $\mathrm{AEs}$ were reported in the study case report form and sent to the HKU/HA HKW IRB.

\section{Statistical analysis}

The main outcome of this study was the proportion of subjects with chemotherapy-induced dermatologic toxicity as measured by NCI CTCAE specific grade $\geq 2$. According to previous researches, the incidence of NCI CTCAE specific grade $\geq 2$ after the intervention was approximately $30 \%$ (11). Our preliminary study showed that the proportion of subjects with NCI CTCAE specific grade $\geq 2$ after taking the $\mathrm{YH} 0618$ decoction decreased to $10 \%$; whereas, difference in proportion of subjects with skin toxicity examined by Z-test between the YH0618 group and control group was $20 \%$. In order to achieve a type I error alpha of 0.05 and power of $90 \%$, the minimal number of people need in each group was 82 . Considering a $20 \%$ attrition rate at the end of follow-up, therefore, at least 107

subjects in each group (a total of 214) was planned for this study.

The collected data were entered into an Excel file by two students and analyzed by a statistician with Statistical Product and Service Solutions (SPSS) version 22.0. Proofreading of the data was carried out and any error due to data entry was corrected before data analysis. Analyses were performed based on intention-to-treat (ITT) principles, and any missing data in the follow up visits were supplemented using multiple imputation. The baseline demographics and clinical characteristics were summarized by descriptive statistics. Missing values at follow up visits or drop outs were evaluated to identify any potential bias. For the primary outcomes, relative risk for the incidence of dermatologic toxicities with grade $\geq 2$ obtained by normal approximation to the binomial distribution was analyzed. Independent sample $t$ tests were adopted to compare the changes in nail color and skin color from baseline to 6 weeks between the two groups. Since participants were randomly divided into the intervention group or waiting list group, little confounding is expected. However, potential confounding variables were those that differ between groups at baseline and were significantly correlated with outcomes. In case any potential confounders identified, logistic regression model and multiple linear regression model were used to analyze the binary and continuous outcomes respectively. 
The outcomes at all time points were assessed in repeated measures mixed models, using ANCOVA model fitting terms of group. Baseline values were included in the model as a covariate. Change from baseline data at week 3, week 6 and week 12 was analyzed separately. Change from baseline for the incidence of dermatologic toxicities with grade $\geq 2$, multinomial logistic regression was performed to assess the group as a relative risk. For the categorical variables, Chisquare test or Fisher exact test was used. For the continuous variable, a simple $t$-test was performed.

\section{Results}

\section{Participants}

Between July 2015 and September 2017, a total of 405 patients were screened and 214 eligible participants provided written informed consents. Of these 214 patients recruited to the study, 104 were allocated to YH0618 decoction group and 110 to wait-list control group. Participants who completed the second assessment were evaluable for the primary end point and composed the ITT population (Figure 4).

Baseline demographic data and clinical characteristics in each group were summarized in Table 1. Of the study, the mean age of participants was 51.5 years old (median $=52.0$, range $=25-75)$. The majority were females $(93 \%)$ and married (76.4\%), living with family members $(92.5 \%)$, had obtained senior high school education or higher (70.3\%), and no need to work (87.0\%). Most of the patients were breast cancer (76.6\%). Both groups were similar at baseline, except for the education level $(\mathrm{P}=0.037)$ and cancer stage $(\mathrm{P}=0.015)$.

\section{Variations of other treatments}

Comparison of western standard treatments and possible detoxification treatments between groups at different time points were shown in Table 2. At the time of baseline assessment, 27\% of the patients in the YH0618 group and $19 \%$ of the patients in the wait-list control group reported that they were receiving other standard treatments, such as radiotherapy and targeted therapy. With the requirement of treatments, the ratio of receiving standard treatments increased in both groups at 3-, 6- and 12-week. However, no significant differences were observed between groups at every assessment point $(\mathrm{P}>0.05)$. As for other possible detoxification therapy, $13.5 \%$ of the patients in YH0618 group and $9 \%$ of the patients in wait-list control group adopted Chinese herbal medicine or acupuncture or sports at baseline. During first 6-week intervention, patients who chose other possible detoxification treatments in YH0618 group kept stable (baseline, 13.5\%; 3-week, 11.2\%; 6-week, $15.7 \%)$. While, in the wait-list group, patients attempted to receive other possible detoxification treatments had been increasing (baseline, 9\%; 3-week, 16.4\%; 6-week, 21.1\%) as they would like to expel toxins from their body as soon as possible. However, during the last 6 weeks, $28.1 \%$ of patients in YH0618 group and 20\% patients in wait-list group received other possible detoxification treatments, because the patients in the $\mathrm{YH} 0618$ group stopped receiving decoction, while the patients in the wait-list group began to receive the YH0618 soup. Although some patients received other possible detoxification treatments during the clinical trial, there were no significant differences between groups at every assessment point $(\mathrm{P}>0.05)$.

\section{Primary outcome}

There were no significant differences in the incidence of all skin toxicities items $\geq$ grade 2 between two groups at baseline $(\mathrm{P}>0.05)$. However, after 6 -week intervention (the primary efficacy analysis time point), YH0618 significantly reduced the average incidence of skin toxicities $\geq$ grade $2(\mathrm{P}=0.0127)$. Significant differences were also showed in terms of alopecia (OR, 0.776; 95\% CI, 0.6-1.0; $\mathrm{P}=0.048)$ and nail discoloration (OR, 0.653; $95 \%$ CI, 0.5-0.9; $\mathrm{P}=0.005)$ between two groups. Grade 2 or higher for alopecia and nail discoloration were reported in $49.4 \%, 40.4 \%$ of patients in the YH0618 group and $60 \%, 50.3 \%$ of patients in the wait-list control group, respectively. Besides, YH0618 improved chemotherapyinduced skin hyperpigmentation in some degree, although there was significant difference. The incidence of skin hyperpigmentation grade $\geq 2$ after 6 -week was $5.6 \%$ in YH0618 treatment group, and $13.0 \%$ in the wait-list group, respectively $(\mathrm{P}=0.084)$. The results at baseline and primary outcome assessment point were shown in Table 3.

Table 4 depicted the effects of YH0618 on the values of the $\mathrm{L}^{*} \mathrm{a}^{*} \mathrm{~b}^{*}$. At 6-week, significant differences between two groups were observed for the $\mathrm{a}^{*}$ and $\mathrm{b}^{*}$ values of forehead ( $\mathrm{a}^{*}$ : 95\% CI, 0.8-2.8; P=0.001; b*: 95\% CI, 0.4-3.8; P =0.017), left face (a*: 95\% CI, 0.3-2.5; $\mathrm{P}=0.01 ; \mathrm{b}^{*}$ : 95\% CI, 1.0-4.1; $\left.\mathrm{b}^{*}=0.002\right)$ and right face $\left(\mathrm{a}^{*}: 95 \% \mathrm{CI}, 0.1-2.2 ; \mathrm{P}=0.027 ; \mathrm{b}^{*}\right.$ : 


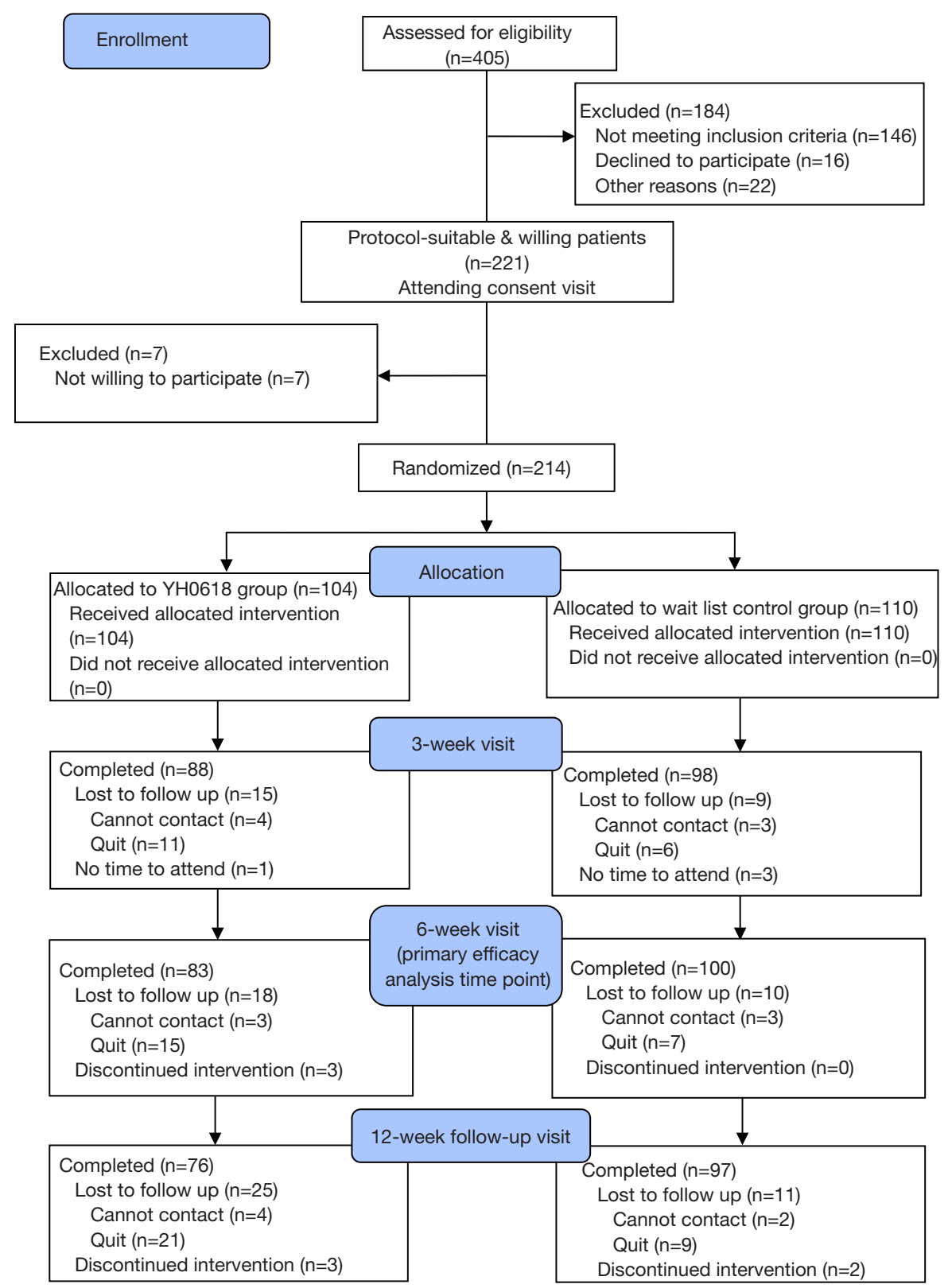

Figure 4 CONSORT diagram of recruitment, randomization and participants.

95\% CI, 0.8-3.8; $\mathrm{P}=0.003)$. Significant differences were also observed in the $L^{*} a^{*} b^{*}$ values of right thumb $\left(L^{*}: 95 \%\right.$ CI, 1.4-6.0; $\mathrm{P}=0.002 ; \mathrm{a}^{*}$ : $95 \% \mathrm{CI},-3.5$ to $-0.39 ; \mathrm{P}=0.015$; $b^{*}$ : $95 \% \mathrm{CI},-4.1$ to $\left.-0.9 ; \mathrm{P}=0.002\right)$, for the $\mathrm{a}^{*}$ values of lower jaw (95\% CI, 0.2 to $2.5 ; \mathrm{P}=0.018$ ). The appearance of hair, hand and nail changes in YH0618 group and waitlist control group at baseline and at 6-week were showed in Figure 5 .

\section{Secondary outcome}

No particularly severe dermatologic toxicity with grade 4 (moderately severe) or 5 (severe) were reported over the whole study. The incidence of specific grade $\geq 2$ dermatologic toxicities at 3- and 12-week were showed in Tables 5,6. No significant differences were presented between groups at both 3 -week and 12-week. 
Table 1 Baseline demographics and clinical characteristics

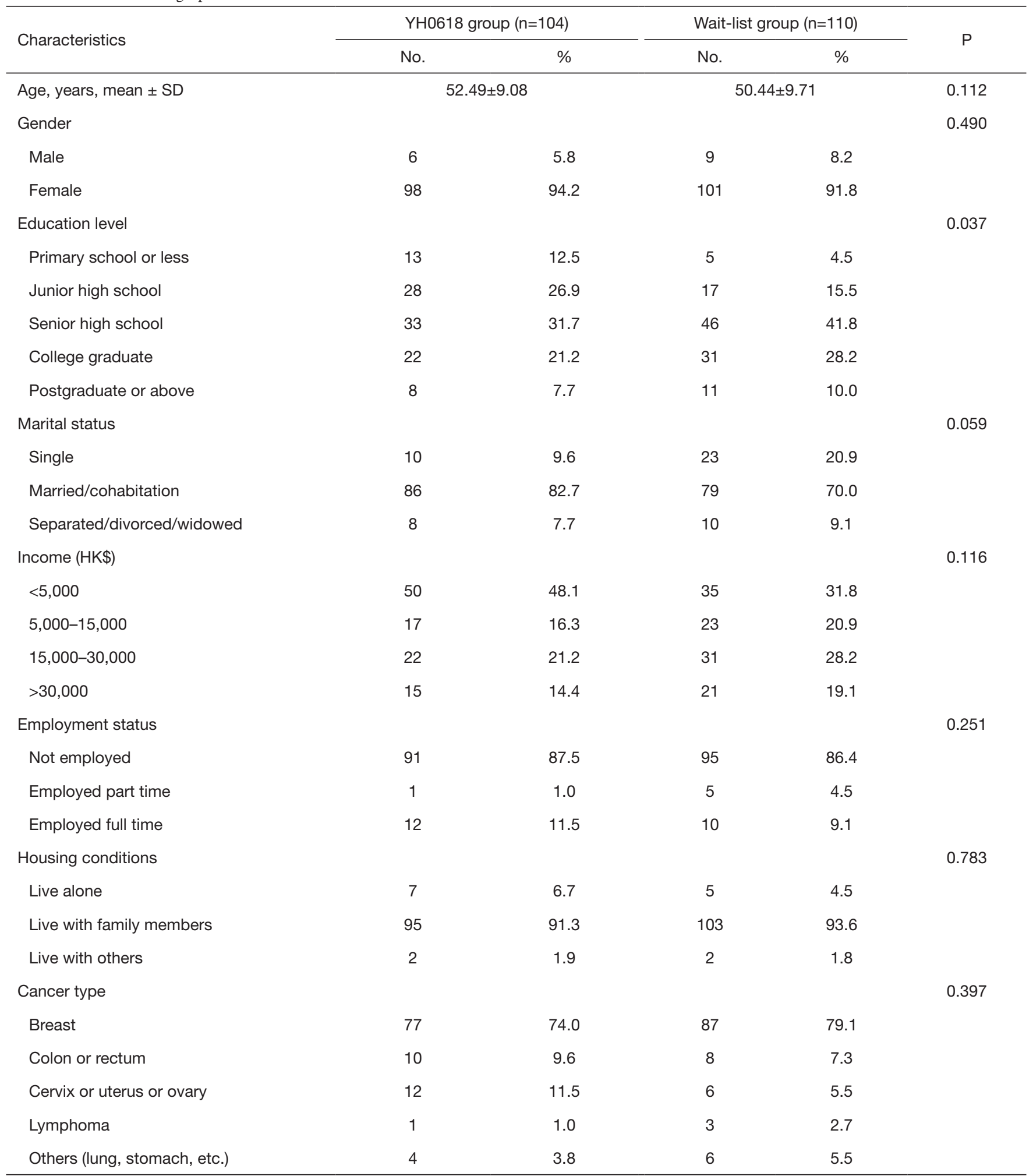

Table 1 (continued) 
Table 1 (continued)

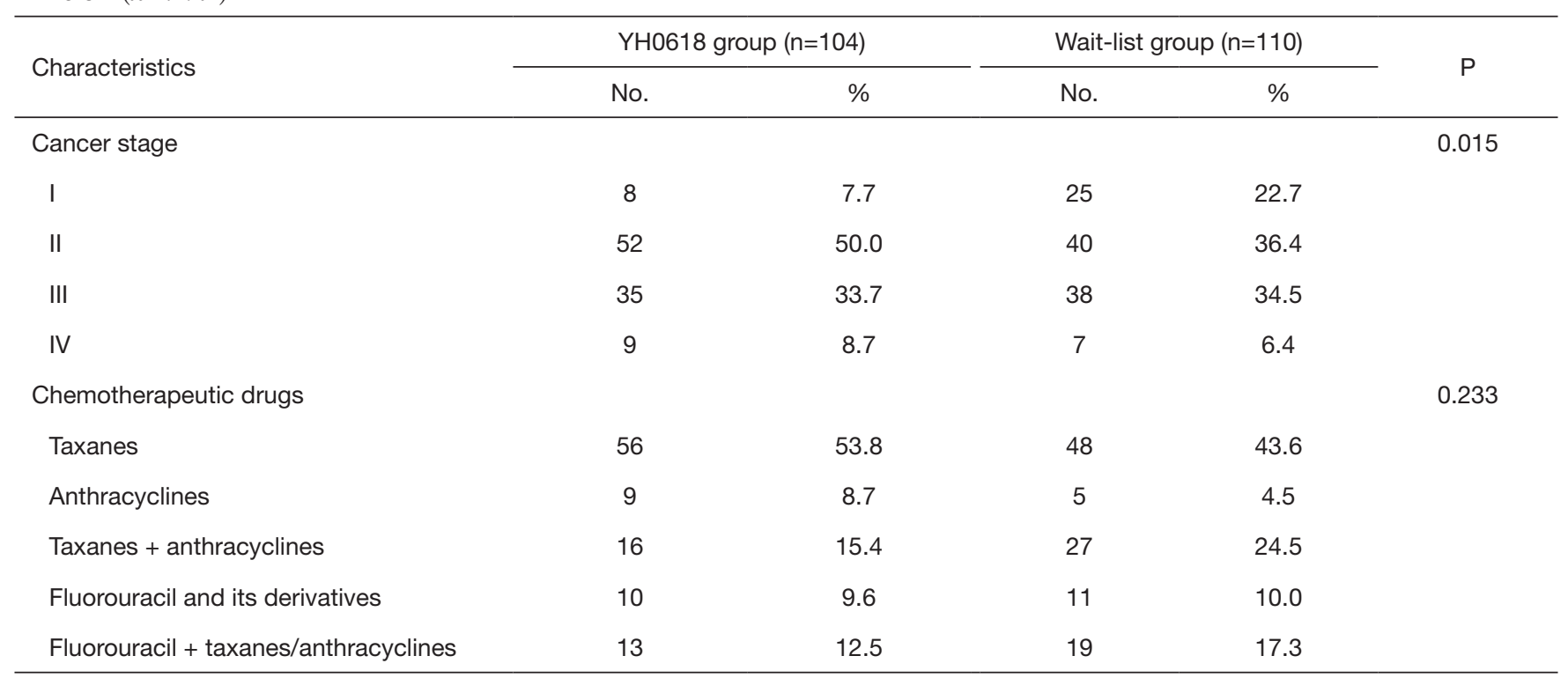

$\mathrm{P}$ values were calculated by independent $t$-test and $\chi^{2}$ tests. A two-sided $\mathrm{P}<0.05$ indicated statistical significance between two groups.

For all patients, it was uneventful to obtain image and data. $L^{*} a^{*} b^{*}$ values of face skin, hands and nails in YH0618 group and wait-list control group from baseline to 12 -week were presented in Figure 6. Except for the $\mathrm{b}^{*}$ value of right thumb $(\mathrm{P}=0.01$, Figure $6 L)$ and $\mathrm{b}^{*}$ value of right middle finger $(\mathrm{P}=0.01$, Figure $6 \mathrm{~N})$, no significant differences of $\mathrm{L}^{*} \mathrm{a}^{*} \mathrm{~b}^{*}$ values were observed between two groups at baseline. At 3-week, YH0618 significantly increased all a* $\mathrm{b}^{*}$ values in four face areas (Figure $6 A, B, C, D$ ) and increased the $\mathrm{b}^{*}$ value of right thumb (Figure $6 L$, all $\mathrm{P}<0.05$ ). At 6 -week, significant differences between two groups were observed for the $\mathrm{a}^{*}$ and $\mathrm{b}^{*}$ values of forehead $\left(\mathrm{P}_{\text {of a }} \mathrm{a}^{*}<0.001\right.$, $\mathrm{P}$ of $\mathrm{b}^{*}=0.003$, Figure $6 A$ ), left face ( $\mathrm{P}$ of $\mathrm{a}^{*}=0.02, \mathrm{P}$ of $\mathrm{b}^{*}<0.001$, Figure $\left.6 B\right)$ and right face $\left(\mathrm{P}\right.$ of $\mathrm{a}^{*}=0.04, \mathrm{P}$ of $\mathrm{b}^{*}=0.001$, Figure $\left.6 C\right)$, for the $\mathrm{a}^{*}$ values of lower jaw $(\mathrm{P}=0.02$, Figure $6 D$ ). Significant differences were also observed in the $\mathrm{L}^{*} \mathrm{a}^{*} \mathrm{~b}^{*}$ values of right thumb (P of $\mathrm{L}^{*}=0.003$, $\mathrm{P}$ of $\mathrm{a}^{*}=0.01, \mathrm{P}$ of $\mathrm{b}^{*}=0.04$, Figure $6 L$ ). There were no significant differences in the color of any skin and nails between two groups at 12-week.

A substantial increase in HGB level occurred over the first 6 weeks (Figure 7), which was greater in the waitlist control arm (mean change level in YH0618 group, $0.66 \mathrm{gm} / \mathrm{dL}$; mean change level in wait-list group, $1.24 \mathrm{gm} / \mathrm{dL} ; \mathrm{P}=0.005)$ and persisted throughout the last 6 weeks period (mean change level in YH0618 group,
$1.12 \mathrm{gm} / \mathrm{dL}$; mean change level in wait-list group, $1.73 \mathrm{gm} / \mathrm{dL} ; \mathrm{P}=0.004$; Figure $7 G$ ). In addition, there were significant differences in creatinine $(\mathrm{P}=0.017$, Figure $7 M)$ and albumin $(\mathrm{P}=0.030$, Figure $7 L)$ between two groups at 6-week. At 12-week, significant difference was observed in BUN between two groups $(\mathrm{P}=0.012$, Figure $7 N)$. The levels of all clinical objective examination items in both groups at 6- and 12-weeks maintained in a normal range.

Regarding QOL and fatigue, no differences were observed between groups at baseline. Both groups showed significant improvement in overall QOL, physical wellbeing, functional well-being and fatigue over time. The fatigue was significantly relieved in the YH0618 group compared with the control group at 6 -week $(\mathrm{P}=0.005)$. During the first 6 weeks, YH0618 improved the overall QOL, all subscales of QOL and fatigue with time. In the wait-list control group, except for the functional wellbeing, other scores of items increased over time, but the improvement degree was lower than YH0618 group. During the last 6 weeks, patients in the wait-list control group began to receive YH0618 decoction and YH0618 speeded up the improvement of QOL and fatigue. As patients in YH0618 group did not received YH0618 decoction, the improvement in QOL and fatigue in this group was not obvious, but the scores of overall QOL, physical well-being, functional well-being and fatigue had been increasing with 


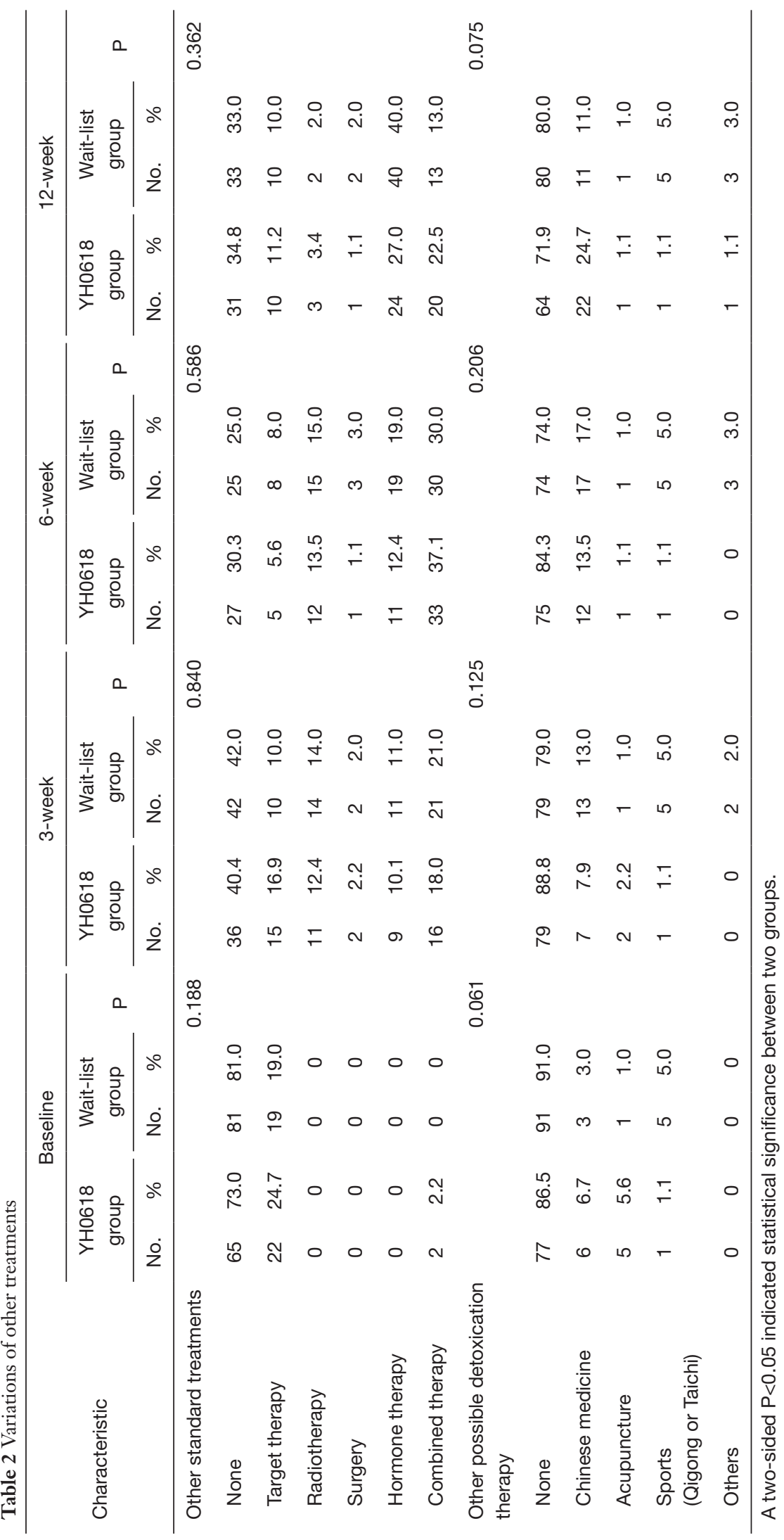


Table 3 Incidence of grade 2 or higher skin toxicities in YH0618 group vs. wait-list control group at baseline and 6-week

\begin{tabular}{|c|c|c|c|c|c|c|c|c|c|c|}
\hline \multirow{2}{*}{ Parameter } & \multicolumn{5}{|c|}{ Baseline (grade 2 or higher) } & \multicolumn{5}{|c|}{ 6-week (grade 2 or higher) } \\
\hline & No. & $\%$ & No. & $\%$ & $P$ & No. & $\%$ & No. & $\%$ & $P$ \\
\hline Dry skin & 35 & 39.3 & 28 & 28.0 & 0.099 & 13 & 14.6 & 15 & 15.0 & 0.939 \\
\hline Pruritus & 16 & 18.0 & 12 & 12.0 & 0.248 & 4 & 4.5 & 6 & 6.0 & 0.644 \\
\hline Rash acneiform & 2 & 2.2 & 3 & 3.0 & 0.748 & 0 & 0 & 0 & 0 & - \\
\hline Rash maculo-papular & 1 & 1.1 & 2 & 2.0 & 0.630 & 0 & 0 & 0 & 0 & - \\
\hline Pain of skin & 4 & 4.5 & 5 & 5.0 & 0.871 & 0 & 0 & 1 & 1.0 & 0.344 \\
\hline Skin hyperpigmentation & 25 & 28.1 & 29 & 29.0 & 0.890 & 5 & 5.6 & 13 & 13.0 & 0.084 \\
\hline Alopecia & 74 & 83.1 & 88 & 88.0 & 0.341 & 44 & 49.4 & 64 & 64.0 & 0.043 \\
\hline Paronychia & 1 & 1.1 & 1 & 1.0 & 0.934 & 0 & 0 & 1 & 1.0 & 0.344 \\
\hline Nail discoloration & 54 & 60.7 & 64 & 64.0 & 0.637 & 36 & 40.4 & 59 & 59.0 & 0.011 \\
\hline Nail ridging & 43 & 48.3 & 51 & 51.0 & 0.712 & 50 & 56.2 & 61 & 61.0 & 0.502 \\
\hline Nail loss & 0 & 0 & 2 & 2.0 & 0.180 & 0 & 0 & 0 & 0 & - \\
\hline
\end{tabular}

$P$ values were calculated by binomial logistic regression. A two-sided $P<0.05$ indicated statistical significance between two groups. Grade of dermatologic toxicity assessment (NCl CTCAE Grading) refers to the severity of the dermatologic toxicity. Any means no symptoms, Mild; asymptomatic or mild symptoms; clinical or diagnostic observations only; intervention not indicated. Grade 2 means moderate; minimal, local or noninvasive intervention indicated; limiting age-appropriate instrumental ADL (refer to preparing meals, shopping for groceries or clothes, using the telephone, managing money, etc.). Grade 3 means severe or medically significant but not immediately lifethreatening; hospitalization or prolongation of hospitalization indicated; disabling; limiting self care ADL (refer to bathing, dressing and undressing, feeding self, using the toilet, taking medications, and not bedridden).

time and the possible reasons were body's self-healing function or durable treatment effect (Figure 8).

\section{AEs}

No severe AEs were reported in this study. Ten events with abdominal bloating may potentially attribute to the YH0618 intervention. However, the $\mathrm{AE}$ was relieved spontaneously after a few days.

\section{Discussion}

A total of 214 patients were randomized to the YH0618 group and the wait-list control group, with 173 patients available for review at the end of the clinical trial. Generally, the overall rate of drop out in clinical trial is designed as $20 \%$, which can guarantee the reliability of the results (12). However, the drop out rate in our protocol was $30 \%$ considering that the patients just completed chemotherapy. Actually, the drop out rate in our clinical trial was less than $20 \%$, therefore, we only recruited 214 patients which could reflect the results.

Statistically significant reductions in the incidence of alopecia and nail discoloration with grade 2 or higher were observed in the YH0618 group compared with the wait-list group after 6-week intervention, demonstrating the success of the YH0618 interventions employed. At baseline, the incidence of alopecia in patients received taxanes and anthracyclines was consistent with prevalence rates $(80-100 \%)$ reported by previous studies (13). Usually, chemotherapy-induced cutaneous reactions are relatively neglected in the research literature and clinical practice, 
Table 4 Nail and skin color in YH0618 group vs. wait-list control group at baseline and 6-week

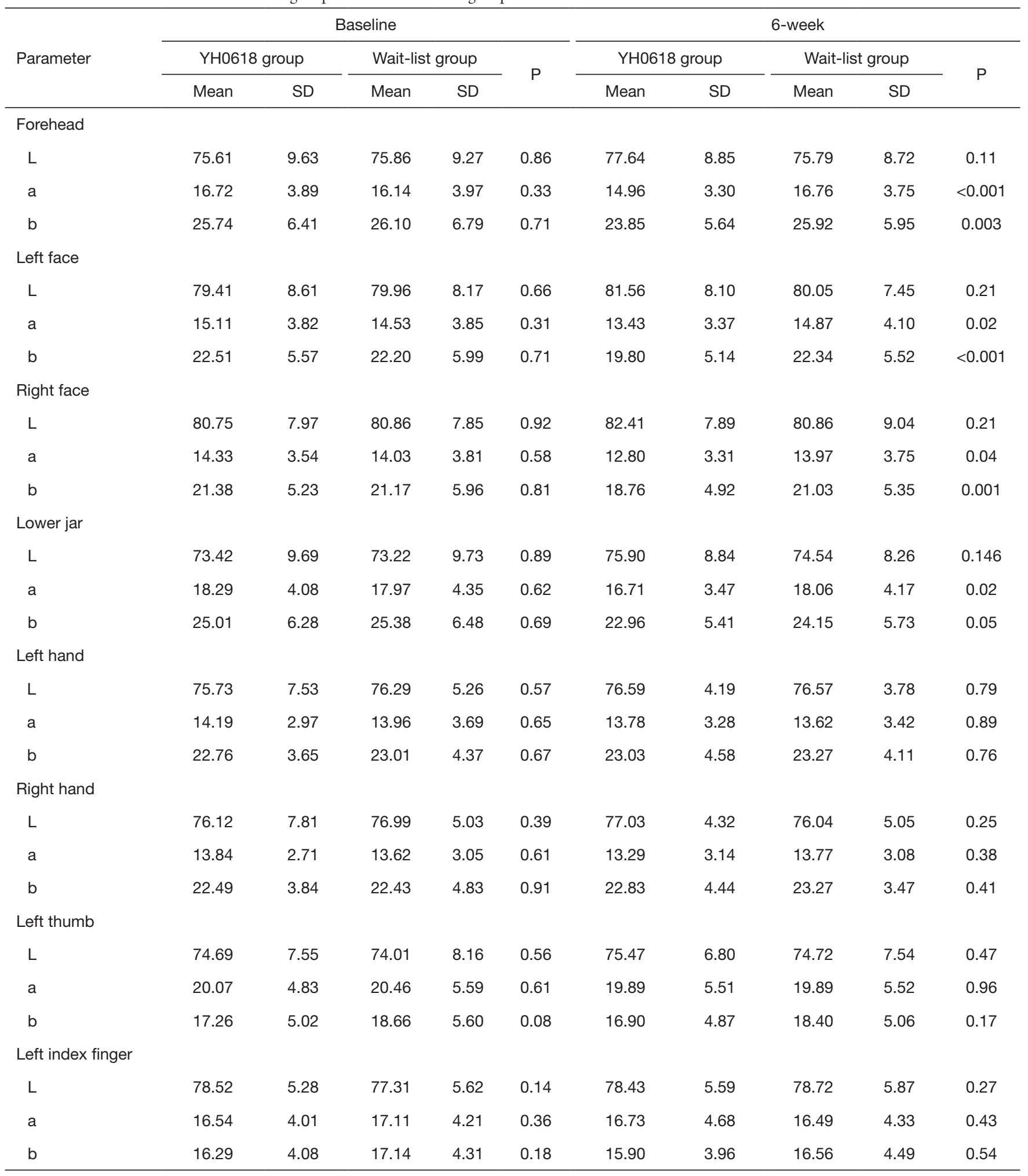

Table 4 (continued) 
Table 4 (continued)

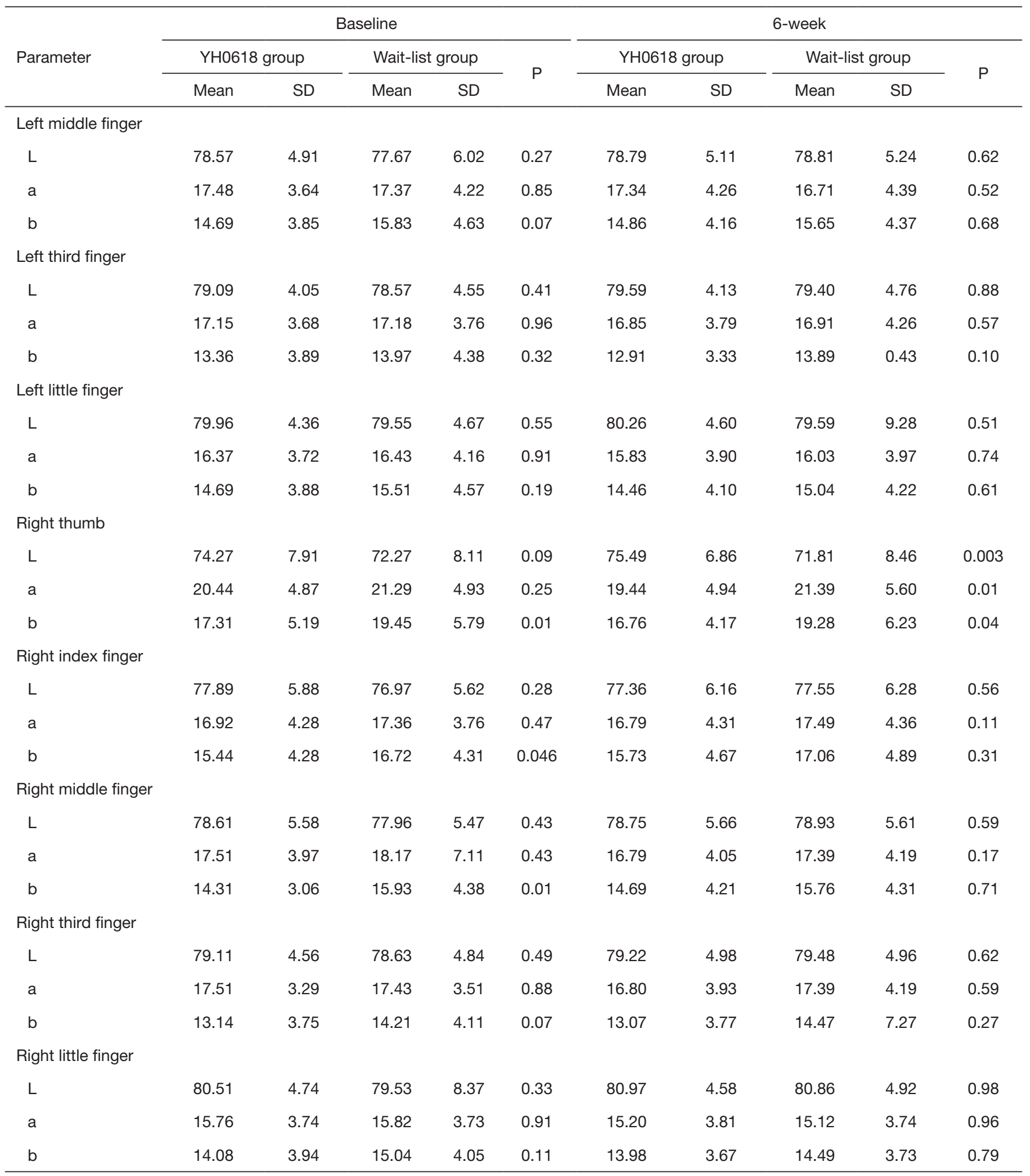

$P$ values were calculated by ANCOVA. A two-sided $P<0.05$ indicated statistical significance between two groups. $L^{*} a^{*} b$ is a "gold standard" for evaluating color. Value $L^{*}$ stands for light/dark, ranging from 0 (black) to 100 (white). Value $a^{\star}$ represents red/green axis, and Value b* represents yellow/blue axis. 


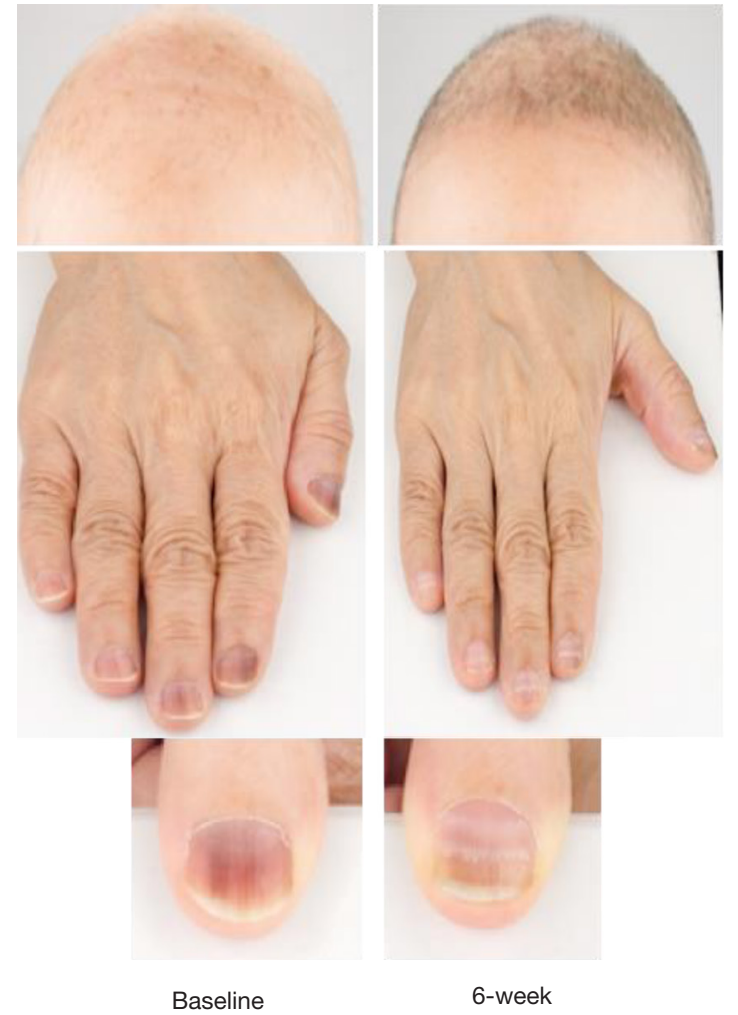

YH0618 group

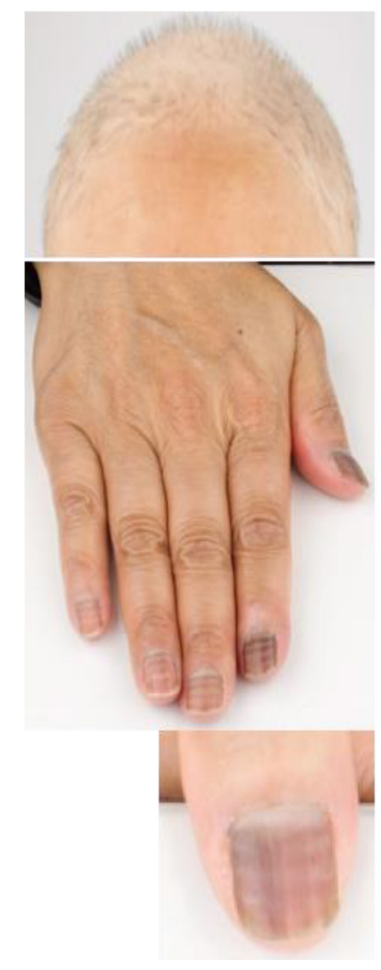

Baseline

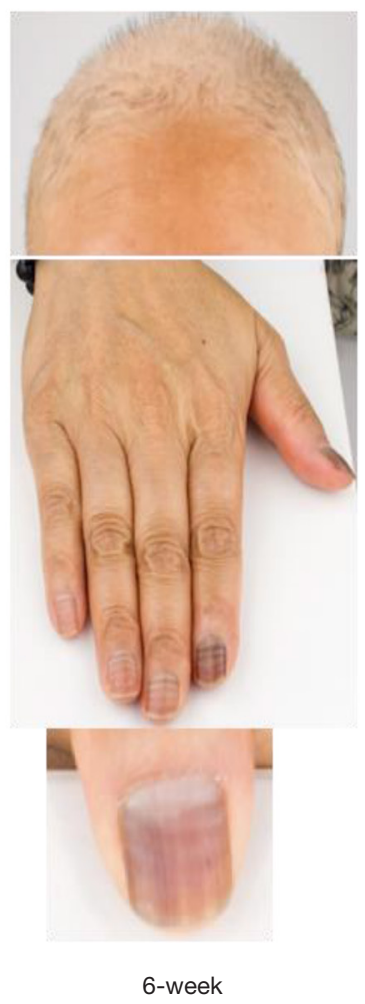

Wait-list control group

Figure 5 The appearance of hair, hand and nail changes.

despite they are common during the course of systemic cancer treatment and sometimes lower patients' QOL and cause functional impairment (14). Recently, some strategies such as cold cap therapy, hair and scalp care, medications, wigs and hairpieces have been used to manage alopecia (15). Unfortunately, none have consistently been shown to be effective. As patients may well tolerate nail discoloration, no treatments are designed to solve the problem. Actually, nail discoloration severely affects beautiful appearance and mood, and is usually associated with skin or mucosal pigmentary changes $(16,17)$. Therefore, YH0618 may be a good choice to ameliorate chemotherapy-induced alopecia and nail discoloration. The encouraging result was supported by other studies investigating the efficacy of black soybean in promoting hair growth and whitening $(18,19)$. Objective $L^{*} a^{*} b^{*}$ values reflected that YH0618 increased brightness, and reduced the red and yellow color for the skin and nails. Some $\mathrm{a}^{*} \mathrm{~b}^{*}$ values in both groups tended to 0 from positive values, but YH0618 increased the process. The phenomenon further demonstrated that the toxins can be expelled from the body via skin, nail, sweat and excrements. However, the significant improvement of discoloration was only improved in the right thumb, and the possible reason was that the color in the right thumb was darker than that of other nails. Such was the fact that the $\mathrm{L}^{*}$ value in the right thumb was lower, and $\mathrm{a}^{*}$ and $b^{*}$ values were higher than others at baseline. Similarity, this phenomenon was also found by Nixon (20) and Issaivanan (21). Besides, another possible reason was subjective bias in photographing and analyzing pictures.

As to other dermatologic toxicities, no significant improvement caused by YH0618 were observed. CIDT are a common and significant complication with the chemotherapy of cancer, limiting both the frequency and intensity of treatment protocols, even lowering the QOL (22). Treatment of CIDT continues to be a challenge because most drugs tested to date have fallen short of providing adequate symptoms relief. To our knowledge, no any accepted management can be used for ameliorating chemotherapy-induced various side effects simultaneously. 
Table 5 Incidence of grade 2 or higher skin toxicities in YH0618 group vs. wait-list group at 3-week

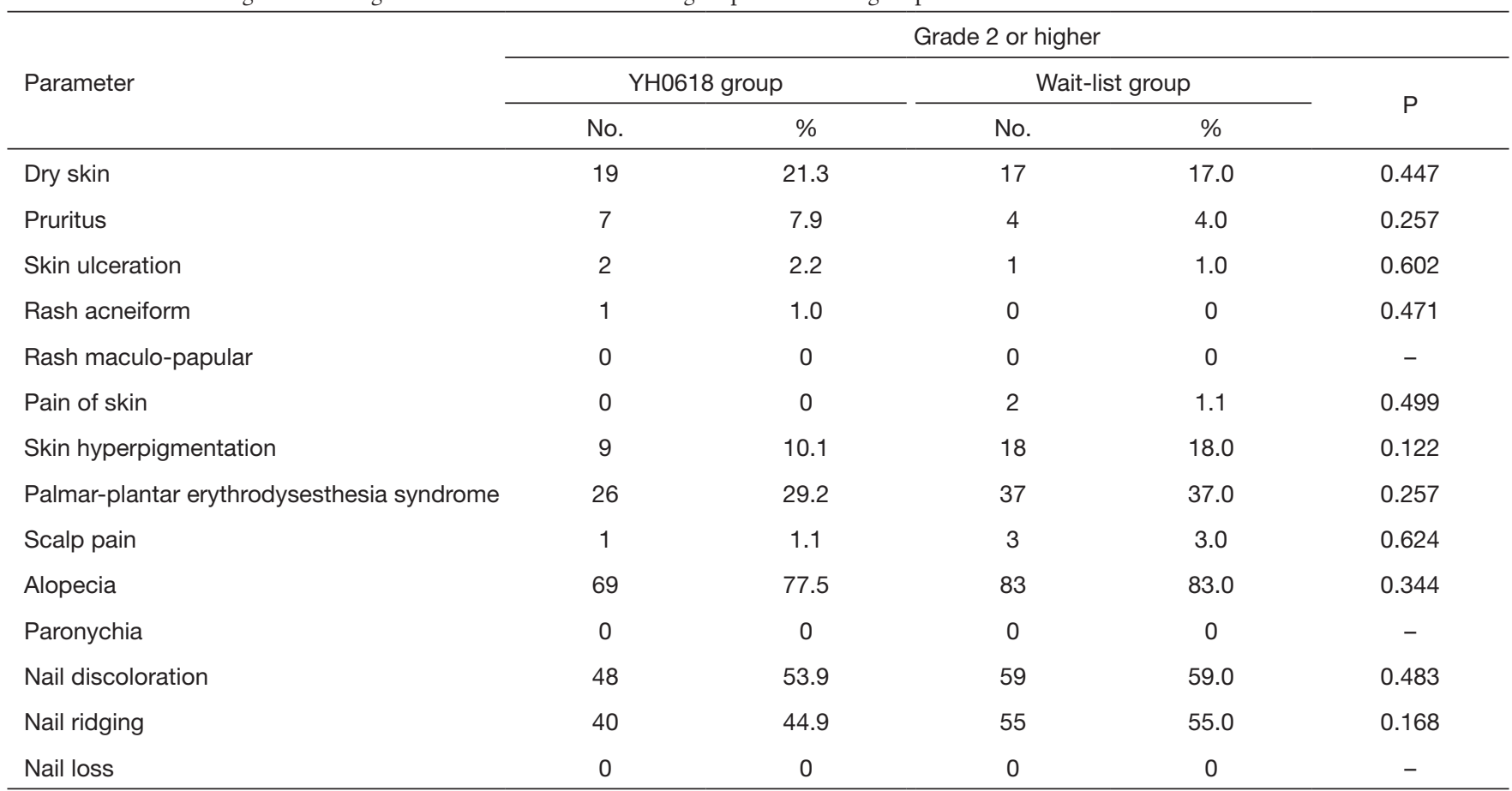

$P$ values were calculated by binomial logistic regression. A two-sided $P<0.05$ indicated statistical significance between two groups. Grade of dermatologic toxicity assessment ( $\mathrm{NCl} \mathrm{CTCAE} \mathrm{Grading)} \mathrm{refers} \mathrm{to} \mathrm{the} \mathrm{severity} \mathrm{of} \mathrm{the} \mathrm{dermatologic} \mathrm{toxicity.} \mathrm{Any} \mathrm{means} \mathrm{no} \mathrm{symptoms,}$ Mild; asymptomatic or mild symptoms; clinical or diagnostic observations only; intervention not indicated. Grade 2 means moderate; minimal, local or noninvasive intervention indicated; limiting age-appropriate instrumental ADL (refer to preparing meals, shopping for groceries or clothes, using the telephone, managing money, etc.). Grade 3 means severe or medically significant but not immediately lifethreatening; hospitalization or prolongation of hospitalization indicated; disabling; limiting self care ADL (refer to bathing, dressing and undressing, feeding self, using the toilet, taking medications, and not bedridden).

Current treatment is geared toward treating only one types of toxicity, such as alopecia, pain, and is limited by significant side effects and expense.

In order to assess these patients' QOL and the YH0618 impact in this study, FACT-G questionnaire was employed. There is evidence that QOL is impaired in patients with chemotherapy $(23,24)$, while Chinese Medicine, foods and dietary supplements such as Ginseng Radix, Radix Astragali, Radix Codonopsis, vitamins, soybeans and $\omega-3$ fatty acid have shown to be effective in improving it $(25,26)$. In this study, although no significant differences in QOL were observed between two groups, YH0618 speeded up the improvement of QOL. Many researches explored the association of chemotherapy-induced alopecia and QOL among women with breast cancer, but very little quantitative data demonstrated the effects of alopecia in the aspects of QOL, except of emotion (27). This study found that YH0618 could improve chemotherapy-induced alopecia without influencing QOL. Chemotherapy-induced fatigue, different from acute fatigue, cannot fully relieved by rest (28). Interestingly, although QOL in these patients was not affected significantly by YH0618, significant alleviation in fatigue were observed in the YH0618 group compared with the wait-list control group after 6-week intervention. A large amount of clinical trials found that above $80 \%$ patients experienced chemotherapy felt that their fatigue had changed their daily routine (29). Patients with fatigue reported significant impairment in completing a variety of activities of daily living, and often presented negative emotion and digressive QOL (30). In this study, last 6-week results obviously demonstrated the link between fatigue and QOL.

The clinical trial was single-blinded, with the assessor and statistician unaware of the treatment allocation for patients. This avoided potential subjective bias towards expected results. Patients were randomly divided into 
Table 6 Incidence of grade 2 or higher skin toxicities in YH0618 group vs. wait-list group at 12-week

\begin{tabular}{|c|c|c|c|c|c|}
\hline \multirow{3}{*}{ Parameter } & \multicolumn{5}{|c|}{ Grade 2 or higher } \\
\hline & \multicolumn{2}{|c|}{ YH0618 group } & \multicolumn{2}{|c|}{ Wait-list group } & \multirow{2}{*}{$P$} \\
\hline & No. & $\%$ & No. & $\%$ & \\
\hline Dry skin & 12 & 13.5 & 10 & 10.0 & 0.456 \\
\hline Pruritus & 1 & 1.1 & 2 & 2.0 & 0.630 \\
\hline Rash acneiform & 0 & 0 & 0 & 0 & - \\
\hline Rash maculo-papular & 0 & 0 & 1 & 1.0 & 0.344 \\
\hline Pain of skin & 0 & 0 & 0 & 0 & - \\
\hline Skin hyperpigmentation & 2 & 2.2 & 4 & 4.0 & 0.686 \\
\hline Alopecia & 6 & 6.7 & 3 & 3.0 & 0.310 \\
\hline Paronychia & 0 & 0 & 0 & 0 & - \\
\hline Nail discoloration & 21 & 23.6 & 25 & 25.0 & 0.822 \\
\hline Nail ridging & 33 & 37.1 & 41 & 41.0 & 0.581 \\
\hline Nail loss & 0 & 0 & 1 & 1.0 & 0.344 \\
\hline
\end{tabular}

$P$ values were calculated by binomial logistic regression. A two-sided $P<0.05$ indicated statistical significance between two groups. Grade of dermatologic toxicity assessment (NCI CTCAE Grading) refers to the severity of the dermatologic toxicity. Any means no symptoms, Mild; asymptomatic or mild symptoms; clinical or diagnostic observations only; intervention not indicated. Grade 2 means moderate; minimal, local or noninvasive intervention indicated; limiting age-appropriate instrumental ADL (refer to preparing meals, shopping for groceries or clothes, using the telephone, managing money, etc.). Grade 3 means severe or medically significant but not immediately lifethreatening; hospitalization or prolongation of hospitalization indicated; disabling; limiting self care ADL (refer to bathing, dressing and undressing, feeding self, using the toilet, taking medications, and not bedridden).

the two groups in order to avoid selection bias. Without a placebo group to compare against was not only due to economic cost, but also to minimize the false negative or positive result caused by placebo effect, because this was the first RCT to evaluate the efficacy of YH0618 in ameliorating CIT. If the result compared with wait-list control group in this study was positive, a placebo will may be adopted in the future. However, the placebo effect has been controversial and Richard Cabot pointed out that placebo should be avoided as it is deceptive (31).

\section{Conclusions}

Overall, this clinical trial demonstrated the efficacy of YH0618 on ameliorating chemotherapy-induced dermatologic toxicity especially nail discoloration, alopecia and skin hyperpigmentation, and on improving fatigue simultaneously with no serious AEs. When considering more and more cancer patients are in face of chemotherapyinduced toxicity, we hope that the study may provide a direct and novel idea that detoxification should become an important part in standard cancer treatments. 


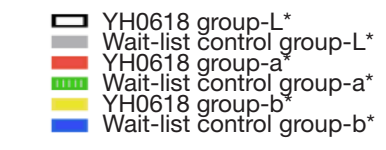

A

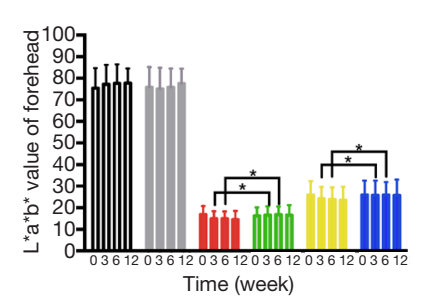

$E$

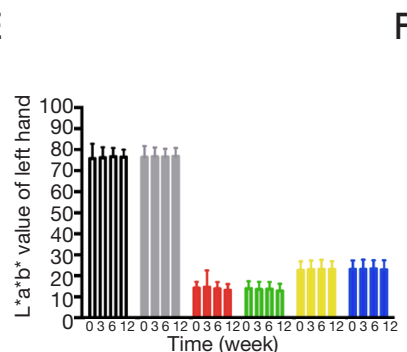

I

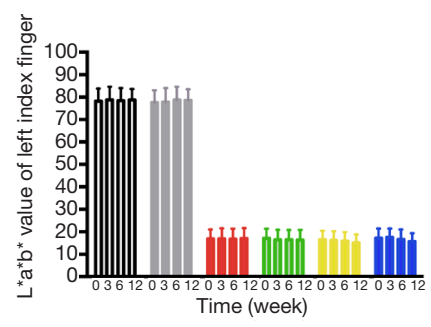

M

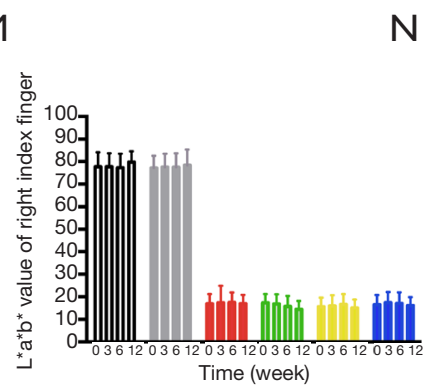

B

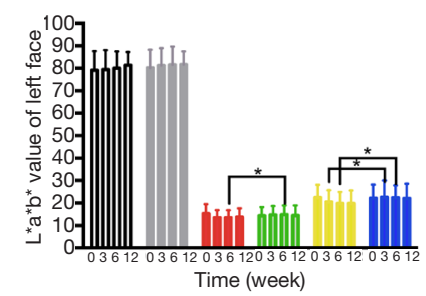

$\mathrm{F}$

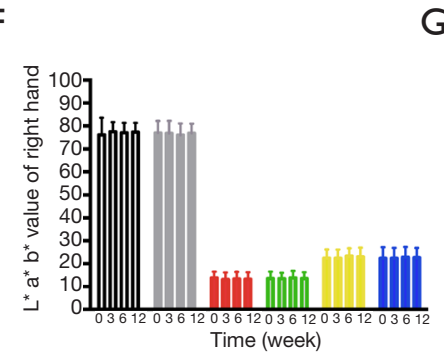

J

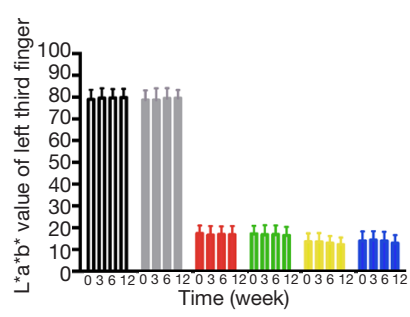

$N$

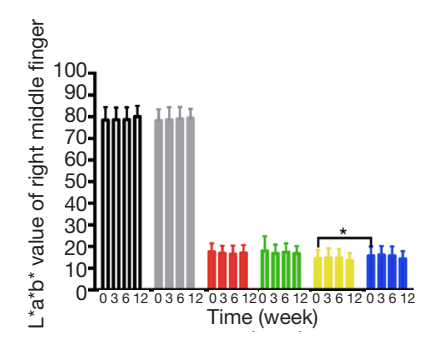

K
C

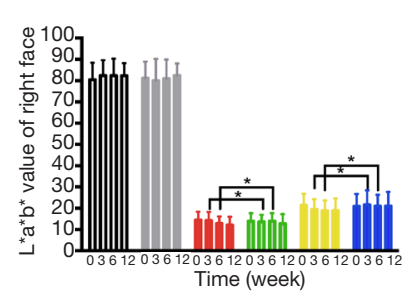

G
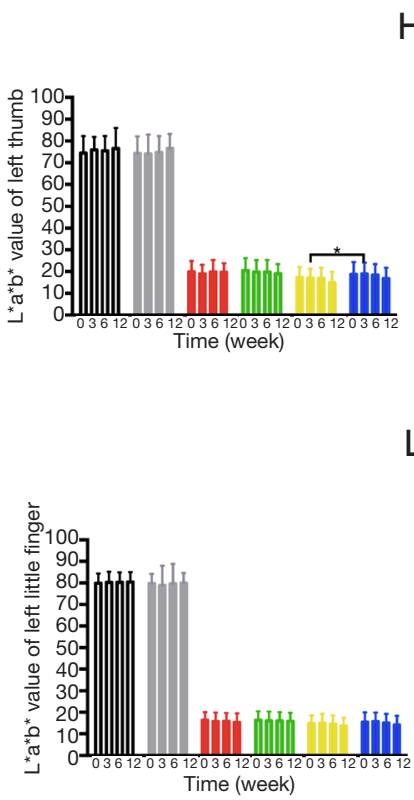

$\mathrm{L}$

$P$

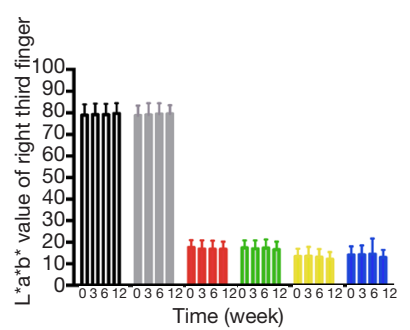

$\mathrm{H}$
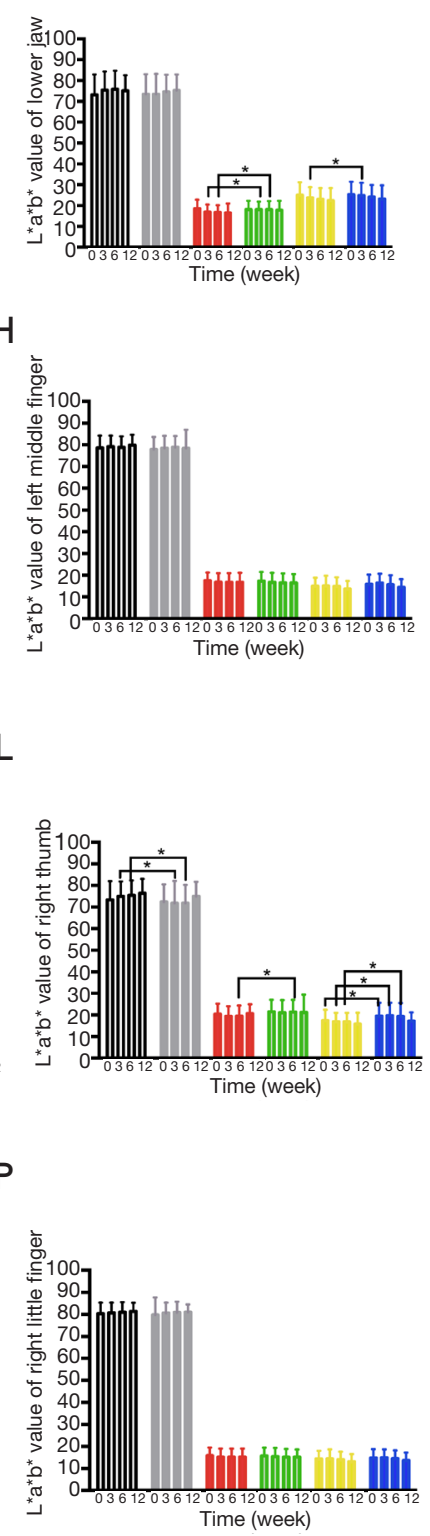

Figure 6 Nail color and skin color in YH0618 group vs. wait-list control group over time. Value $L^{*}$ represents light/dark and extends from 0 (black) to 100 (white). Value $\mathrm{a}^{*}$ represents red/green axis $(-128$ to +127$)$, with green at negative and red at positive $\mathrm{a}^{*}$ values. Value $\mathrm{b}^{*}$ represents yellow/blue axis $(-128$ to +127$)$, with blue at negative and yellow at positive $b^{*}$ values. $\mathrm{P}$ values were calculated by ANCOVA. *Indicated two-sided statistical significance at 0.05 level between two groups. 
$\multimap$ YH0618 group

$\rightarrow$ Wait-list control group

A

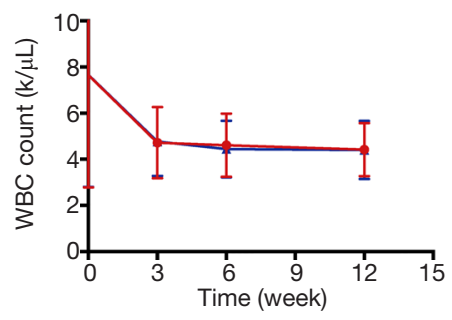

E

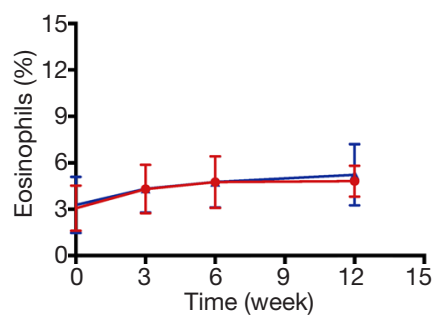

I

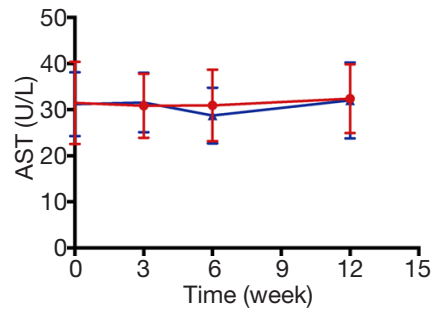

M

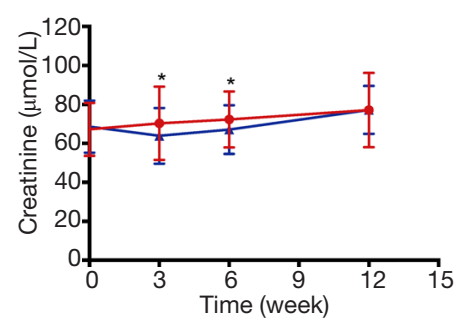

B

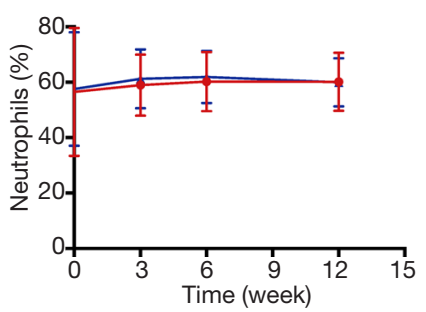

F

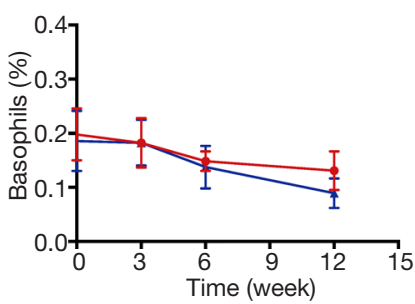

J

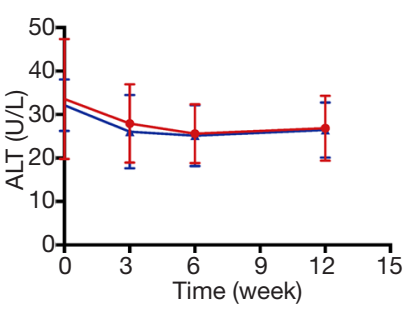

N

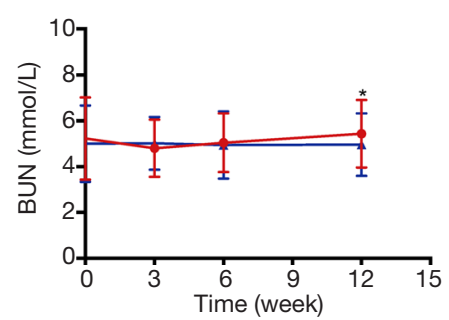

C

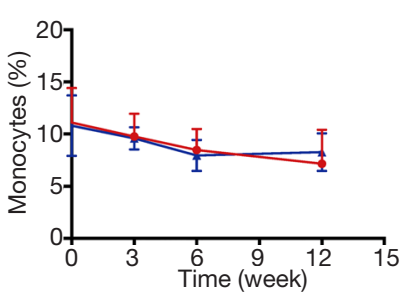

G

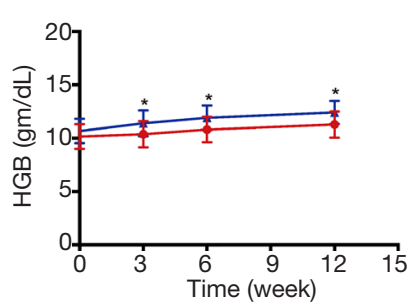

K
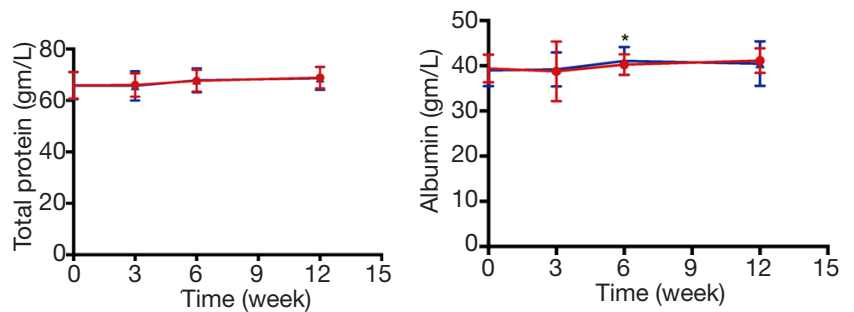

Figure 7 Clinical biomarkers of myelosuppression, hepatotoxicity and nephrotoxicity in YH0618 group vs. wait-list group over time. P values were calculated by ANCOVA. *Indicated two-sided statistical significance at 0.05 level between two groups. 

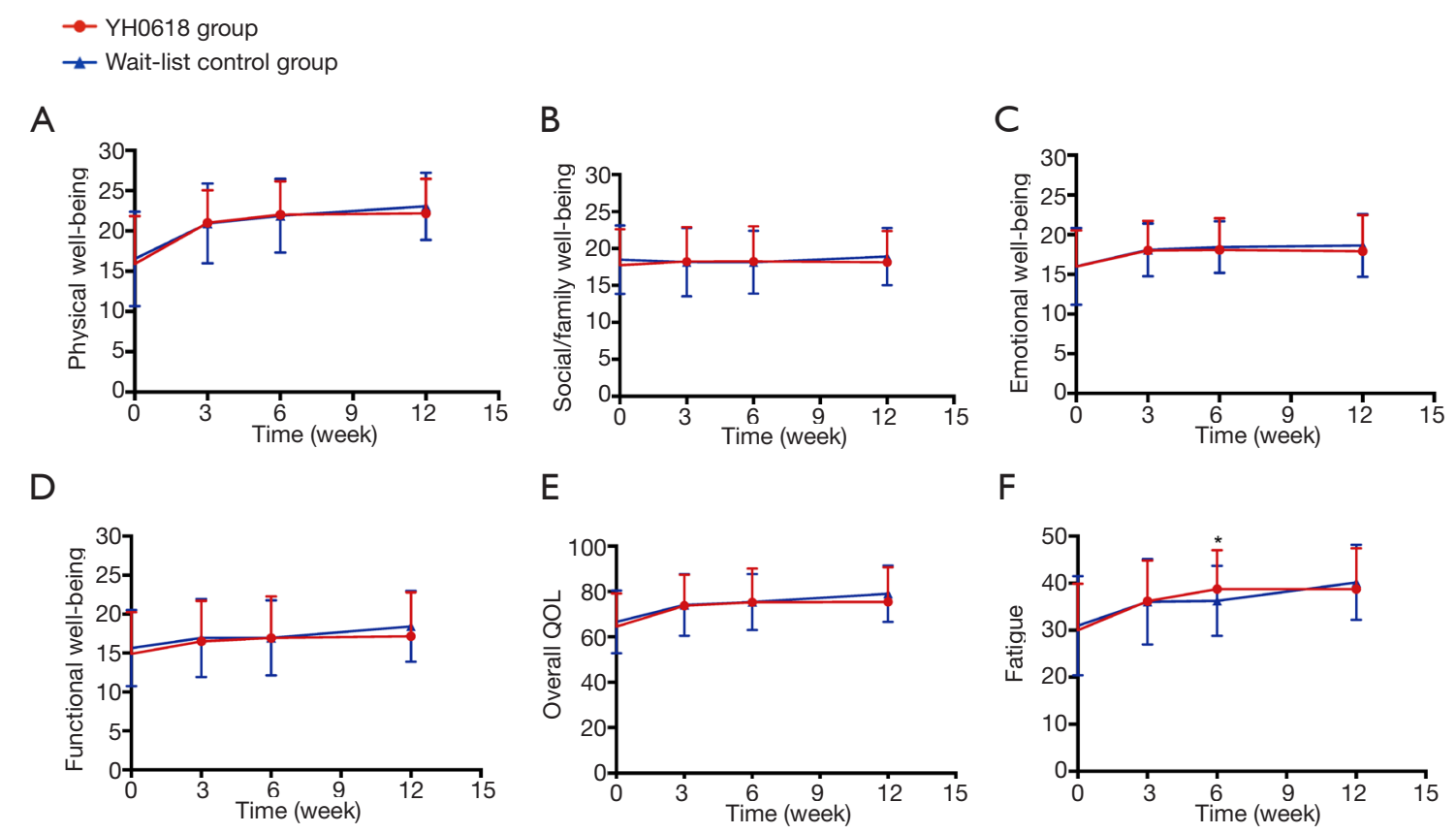

Figure 8 Quality of life and fatigue in YH0618 group vs. wait-list group over time. Higher scores mean better general QOL and less fatigue. $\mathrm{P}$ values were calculated by ANCOVA. *Indicated two-sided statistical significance at 0.05 level between two groups.

\section{Acknowledgments}

The authors would like to thank all patients who consented to be screened and who participated in the study. We are grateful to our colleagues and research staff who participated in the enrollment and preparation of materials of YH0618. The authors also thank co-applicants for their contributions to the study design and trial management and thank assessors and research assistants for their contributions to the completion of this trial.

Funding: This work was supported by Seed Fund for Translational and Applied Research from HKU (201611160019); Innovation and Technology Fund from Hong Kong government (UIM/276); And Postdoctoral Fund from China Postdoctoral Science Foundation (2018M633037); and study on key technology of caulis spatholobi industrialization (No. AB16450008 important international cooperation fund).

\section{Footnote}

Reporting Checklist: The authors have completed the CONSORT reporting checklist. Available at http://dx.doi. org/10.21037/atm-20-5181
Data Sharing Statement: Available at http://dx.doi, org/10.21037/atm-20-5181

Conflicts of Interest: All authors have completed the ICMJE uniform disclosure form (available at http://dx.doi. org/10.21037/atm-20-5181). JY and JC have a patent null licensed to Jianping Chen, Jieshu You, Fei Gao. The other authors have no conflicts of interest to declare.

Ethical Statement: The authors are accountable for all aspects of the work in ensuring that questions related to the accuracy or integrity of any part of the work are appropriately investigated and resolved. The study was conducted in accordance with the Declaration of Helsinki (as revised in 2013). The study was approved by the Institutional Review Board of the University of Hong Kong/ Hospital Authority Hong Kong West Cluster (HKU/ HA HKW IRB) (No.: UW 15-023) and informed consent was taken from all the patients.

Open Access Statement: This is an Open Access article distributed in accordance with the Creative Commons Attribution-NonCommercial-NoDerivs 4.0 International 
License (CC BY-NC-ND 4.0), which permits the noncommercial replication and distribution of the article with the strict proviso that no changes or edits are made and the original work is properly cited (including links to both the formal publication through the relevant DOI and the license). See: https://creativecommons.org/licenses/by-nc-nd/4.0/.

\section{References}

1. Heidary N, Naik H, Burgin S. Chemotherapeutic agents and the skin: An update. J Am Acad Dermatol 2008;58:545-70.

2. Miller KK, Gorcey L, McLellan BN. Chemotherapyinduced hand-foot syndrome and nail changes: a review of clinical presentation, etiology, pathogenesis, and management. J Am Acad Dermatol 2014;71:787-94.

3. Abushullaih S, Saad ED, Munsell M, et al. Incidence and severity of hand-foot syndrome in colorectal cancer patients treated with capecitabine: a single-institution experience. Cancer Invest2002;20:3-10.

4. Lacouture ME, Mitchell EP, Piperdi B, et al. Skin toxicity evaluation protocol with panitumumab (STEPP), a phase II, open-label, randomized trial evaluating the impact of a pre-Emptive Skin treatment regimen on skin toxicities and quality of life in patients with metastatic colorectal cancer. J Clin Oncol2010;28:1351-7.

5. You J, Gao F, Tang H, et al. A medicinal and edible formula YH0618 ameliorates the toxicity induced by Doxorubicin via regulating the expression of $\mathrm{Bax} / \mathrm{Bcl}-2$ and FOXO4. J Cancer 2019;10:3665-77.

6. You JS, Chen JP, Chan JS, et al. Effect of YH0618 soup on chemotherapy-induced toxicity in patients with cancer who have completed chemotherapy: study protocol for a randomized controlled trial. Trials 2016;17:354.

7. Park SB, Goldstein D, Lin CS, et al. Acute abnormalities of sensory nerve function associated with oxaliplatininduced neurotoxicity. J Clin Oncol 2009;27:1243-9.

8. Connolly C, Fleiss T. A study of efficiency and accuracy in the transformation from RGB to CIELAB color space. IEEE Trans Image Process 1997;6:1046-8.

9. Cortez R, Luna-Vital DA, Margulis D, et al. Natural Pigments: Stabilization Methods of Anthocyanins for Food Applications. Compr Rev Food Sci Food Saf 2017;16:180-98.

10. Cella D, Yount S, Sorensen M, et al. Validation of the Functional Assessment of Chronic Illness Therapy Fatigue Scale relative to other instrumentation in patients with rheumatoid arthritis. J Rheumatol 2005;32:811-9.
11. Balagula Y, Rosen ST, Lacouture ME. The emergence of supportive oncodermatology: the study of dermatologic adverse events to cancer therapies. J Am Acad Dermatol 2011;65:624-35.

12. Orrego H, Blake JE, Blendis LM, et al. Long-term treatment of alcoholic liver disease with propylthiouracil. Part 2: Influence of drop-out rates and of continued alcohol consumption in a clinical trial. J Hepatol 1994;20:343-9.

13. Trüeb RM. Chemotherapy-induced alopecia. Semin Cutan Med Surg 2009;28:11-4.

14. Shin H, Jo SJ, Kim DH, et al. Efficacy of interventions for prevention of chemotherapy-induced alopecia: a systematic review and meta-analysis. Int J Cancer 2015;136:E442-54.

15. West HJ. Chemotherapy-Induced Hair Loss (Alopecia). JAMA Oncol 2017;3:1147.

16. Wasner G, Hilpert F, Baron R, et al. Clinical picture: nail changes secondary to docetaxel. Lancet 2001;357:910.

17. Borecky DJ, Stephenson JJ, Keeling JH, et al. Idarubicininduced pigmentary changes of the nails. Cutis 1997;59:203-4.

18. Lai J, Xin C, Zhao Y, et al. Study of active ingredients in black soybean sprouts and their safety in cosmetic use. Molecules 2012;17:11669-79.

19. Kim SC, Kang JI, Kim MK, et al. The hair growth promoting effect of 4-O-methylhonokiol. Eur J Dermatol 2011;21:1012-4.

20. Nixon DW. Alterations in nail pigment with cancer chemotherapy. Arch Intern Med 1976;136:1117-8.

21. Issaivanan M, Mitu PS, Manisha C, et al. Cutaneous manifestations of hydroxyurea therapy in childhood: case report and review. Pediatr Dermatol 2004;21:124-7.

22. DeSpain JD. Dermatologic toxicity of chemotherapy. Semin Oncol 1992;19:501-7.

23. Arora NK, Gustafson DH, Hawkins RP, et al. Impact of surgery and chemotherapy on the quality of life of younger women with breast carcinoma: a prospective study. Cancer 2001;92:1288-98.

24. Broeckel JA, Jacobsen PB, Balducci L, et al. Quality of life after adjuvant chemotherapy for breast cancer. Breast Cancer Res Treat 2000;62:141-50.

25. Mitsugi K, Nakamura T, Kashiwabara N, et al. Protection against methotrexate toxicity by a soybean protein- and omega-3 fatty acid-containing diet: comparative study with a casein-containing diet. Oncol Rep 2004;12:41-5.

26. Vollbracht C, Schneider B, Leendert V, et al. Intravenous vitamin $\mathrm{C}$ administration improves quality of life in breast cancer patients during chemo-/radiotherapy and aftercare: 
results of a retrospective, multicentre, epidemiological cohort study in Germany. In Vivo 2011;25:983-90.

27. Lemieux J, Maunsell E, Provencher L. Chemotherapyinduced alopecia and effects on quality of life among women with breast cancer: a literature review. Psychooncology 2008;17:317-28.

28. Cella D, Davis K, Breitbart W, et al. Cancer-related fatigue: prevalence of proposed diagnostic criteria in a United States sample of cancer survivors. J Clin Oncol

Cite this article as: You J, He Y, Zhi H, Lee VH, Chan S, Lao L, Liu H, Chen J. Effect of a medicinal and edible decoction YH0618 on chemotherapy-induced dermatologic toxicity: a randomized controlled trial. Ann Transl Med 2021;9(1):4. doi: 10.21037/atm-20-5181
2001;19:3385-91.

29. Koornstra RH, Peters M, Donofrio S, et al. Management of fatigue in patients with cancer -- a practical overview. Cancer Treat Rev 2014;40:791-9.

30. Curt GA. Impact of fatigue on quality of life in oncology patients. Semin Hematol 2000;37:14-7.

31. de Craen AJ, Kaptchuk TJ, Tijssen JG, et al. Placebos and placebo effects in medicine: historical overview. J R Soc Med 1999;92:511-5. 